\title{
Thick-film multi-DOF force / torque sensor for wrist rehabilitation
}

\author{
C. Jacq ${ }^{1}$, B. Lüthi ${ }^{1}$, Th. Maeder ${ }^{1}$, O. Lambercy ${ }^{2}$, R. Gassert ${ }^{2}$ and P.Ryser \\ ${ }^{1}$ Laboratoire de Production Microtechnique (LPM), EPFL, Lausanne, Switzerland \\ ${ }^{2}$ Rehabilitation Engineering Lab, ETH Zurich, Zurich, Switzerland \\ E-mail: caroline.jacq@epfl.ch \\ Presented at Eurosensors XXIII, manuscript \#8426
}

\begin{abstract}
A complete six-degree-of-freedom (6-DOF) force / torque sensor has been designed and fabricated, adapted to wrist rehabilitation applications, with the focus laid on simple, straightforward manufacturing processes. This paper details the mechanical design, 3D modeling, manufacture and characterisation of the sensor.

Compared to previous work [1], this design has the advantage of simple, fully planar machining, and the loadsensing elements all lie on the same plane, making the device compatible with single-film deposition or a foil bonding process. The sensor was machined from steel, and the piezoresistive load-sensing bridges were deposited using thick-film technology. We used commercial thick-film materials in the work described here. A new lead-free materials system compatible with low processing temperatures $\left(<700^{\circ} \mathrm{C}\right)$ will shortly replace the commercial one and is expected to eventually also be adaptable to aluminium substrates. Such a sensor could find applications in multi-DOF therapeutic robotic systems as well as in isometric motor training.
\end{abstract}

Keywords: sensor; 6 DOF; thick-film; rehabilitation application; steel.

\section{Introduction}

Recently, robotic devices allowing the patient to monitor his force / torque level in order to increase muscle strength and control of the impaired limb, and reduce abnormal muscle synergies [2,3] have been increasingly used in post-stroke rehabilitation exercises [4]. In order to perform robot-assisted rehabilitation of individual joint such as the wrist, a force sensor capable of measuring all six degrees of freedom (DOFs) is required. This would allow precise assessment of the joint properties and patient's force/torque capabilities, while allowing the detection of undesired parasitic forces/torques in DOF not controlled by the wrist. This would provide physiotherapists with a quantitative assessment of movement synergies, and allow rehabilitation therapies tailored to the patient's abilities. However, existing designs of 6 DOF force sensors [1] can be quite complex, often requiring complicated machining steps and, even worse, gluing strain gauges in small recesses, resulting in 
expensive and complicated devices. We endeavoured to design a sensor having a planar sensing area compatible with film deposition / foil bonding processes for the strain gauges [5-8]. In contrast to other work [5,6], we aimed here for a simple, rugged and relatively inexpensive device, whose manufacture is compatible with basic 3-axis milling. Such a design has previously been successfully used by the authors to fabricate a ligamentbalancing force sensor for total knee arthroplasty (TKA) $[7,8]$. In this device, the thick-film sensing circuit has been deposited directly onto a steel structure, which is much more rugged than ceramic. This approach was also selected for the present sensor.

In this study, we present the mechanical concept of the 6-DOF force sensor, its fabrication and the characterisation of its response.

\section{Mechanical design}

\subsection{Requirements/specifications}

The precise measurement of interaction forces generated by subjects during robot-assisted rehabilitation is critical to gain a better understanding of movement disorders and potentially correct them by adjusting therapy parameters. For example, resistive forces measured during passive limb motion can be used to quantitatively estimate abnormal muscle tone, e.g. during finger extension, one of the major impairments observed in stroke, the assessment of which is so far limited to objective estimation of physiotherapists. Multi-axis force/torque measurements would also allow monitoring of parasitic forces and torques applied by subjects as a result of compensatory movements, or abnormal movement synergies.

Forces and torques measured along degrees-of-freedom of the sensor that are mechanically constrained by the robotic system can be used to calculate and display virtual error movements based on a model of interaction dynamics (virtual admittance law), providing subjects with precious insight into improved motor coordination [9]. This approach could pave the way for the development of robotic devices with reduced active DOF (Figure 1), promising more compact table-top rehabilitation systems, with reduced complexity and cost, thereby increasing the potential for vast deployment in rehabilitation centres and stroke patients' homes [2].

The specifications for the design of a $6 \mathrm{DOF}$ force/torque sensor to be applied for rehabilitation of the wrist joint are obtained from biomechanical data found in the literature [10,11], and adapted based on our own experience with stroke patients [3,9] (Table 1). Moreover, in order to integrate the sensor onto an existing robotic platform, the diameter of the sensor should preferably not exceed $40 \mathrm{~mm}$ and the height should not exceed $10 \mathrm{~mm}$. Finally the cost must be rather low compared to commercial sensors (3 to $10 \mathrm{k} €$ ). 
In a previous work, we developed a force sensor based on metallic substrate for TKA $[7,8]$. This sensor infers the force (amplitude and localisation) applied by the bones on the implant in order to balance the tension in the lateral ligaments and reduce the wear of the implant. The measured force is a linear combination of three forces detected with three Wheatstone bridges positioned in a triangular geometry. In the design presented in this paper, we have adapted and extended this strategy to measure the three forces and the three moments.

\subsection{Concept}

The sensor consists, in principle, of a fixed outer rim and an inner mobile ring, linked by three symmetrically arranged force-sensing bridges onto which the sensitive materials have been deposited (Figure 2).

To concentrate deformations at precise locations, each bridge has some thinned sections that locally weaken the structure, which concentrates the elastic strains and thus allows piezoresistive sensing (Figure 3). The piezoresistors are deposited onto these thinned zones, and linked electrically in reconfigurable Wheatstone bridges (Figure 4), which allows sequential extraction of two forces (Figure 3): horizontal tension / compression $(\mathrm{H})$ and vertical force $(\mathrm{V})$. This reconfiguration is effected by swapping the positive and negative power supply of one half of each bridge by the means of analogue switches mounted in an H-bridge configuration. Overall, this gives $3 \times 2=6$ forces that, when combined appropriately, allow extraction of the 6 DOFs.

\subsection{Dimensioning of the sensor}

The dimensions have been first calculated roughly, using the values from the specifications (Table 1) and the relations below. The values selected for the sensor are given, together with calculated stresses and the symbols, in Table 2. The effective length of the stressed beam $l$ was taken to be the thinnest section.

- Stress in tension/compression:

- Maximal bending stress:

- Maximum of parallel force

- Maximum of perpendicular force

$$
\begin{aligned}
& \sigma_{/ /}=F_{/ /} /(2 b \cdot h) \\
& \sigma_{b}=F_{\perp} \cdot l \cdot h /(8 I), \text { with } I=b \cdot h^{3} / 12 \\
& F_{/ / \max } \approx 100 \mathrm{~N} \\
& F_{\perp \max }=200 \mathrm{~N} / 3 \approx 70 \mathrm{~N}
\end{aligned}
$$

In addition to the thinned sensing sections, the design of the module has been optimised with additional laterally narrowed zones at the end of the bridges acting as elastic hinges to decouple the shear loads and decrease their effects on the measurement. Due to the space needed by the thick-film conductors, the topside had to be left somewhat wider, resulting in a T-shaped section or those "hinges". 
The dimensioning was based on the forces and torques exerted by the human wrist, which entailed some compromises in sensitivity of some axes, dictated by the simplicity of the device (Tables 1 and 2). The thickness of the three bridges is $0.8 \mathrm{~mm}$ for a width of $3.2 \mathrm{~mm}$ and an effective length of $2.1 \mathrm{~mm}(3.1 \mathrm{~mm}$ with a fillet of $0.5 \mathrm{~mm}$, see Figures 5 and 6 ). The $0.5 \mathrm{~mm}$ fillet radius was introduced to avoid excessive stress concentrations.

The sensor was designed based on the dimensioning parameters, namely the most sensitive $F_{z}$ specification, but also on the design rules for the screen-printing and for the machining processes. The resulting calculated force and torque values are listed in Table 3.

\subsection{Finite-element modelling (FEM)}

Detailed full-3D FEM (ProEngineer) was carried out, applying forces and moments on the inner axis hole and zero-displacement boundary conditions on the outer rim (see Figure 6). Figure 7 shows the resulting distribution of Von Mises stress (colour) and displacement (exagerated), for applied $M_{z}$ torque (a) and $F_{z}$ force (b). In general, the stresses are well localised in the bridges, and low in the inner and outer rims.

For an applied $M_{z}$ torque (Figure $7 \mathrm{a}$, links in tension / compression, similar to $F_{x}$ and $F_{y}$ forces), the stresses tend to concentrate on the top of the T-section hinges rather than in the sensing areas, indicating that - although the levels are acceptable due to the low sensitivity in this mode - these zones should be redesigned in future versions. The highest Von Mises stress with $M_{z}=5 \mathrm{~N} \cdot \mathrm{m}$ torque applied is $53 \mathrm{MPa}$. For an applied vertical $F_{z}$ force (Figure $7 \mathrm{~b}$, links in bending, similar to $M_{x}$ and $M_{y}$ torques), the situation is much more favourable; the highest stresses are localised as expected, under the thinned force-sensing zones, and the maximum Von Mises stress is an acceptable $150 \mathrm{MPa}$. However, this value is still large compared to the stress experienced on the sensing zones, ca. $90 \mathrm{MPa}$, which indicates the fillet radius should be somewhat increased to reduce the stress concentrations.

\section{Manufacture and characterisation}

\subsection{Metal structure and thick-film process}

The sensor metal body was machined out of a $68 \mathrm{~mm}$ diameter circular slab of standard austenitic stainless steel (1.4301 / AISI 304), using a shaped end mill to ensure $0.5 \mathrm{~mm}$ fillets and thus avoid excessive stress concentrations.

The thick-film materials system (dielectric, conductor and piezoresistive resistor) was applied by screenprinting. As for the previous work in the field, this paper presents results for the sensor instrumented (Figure 8) 
with a commercial high-firing (peak: 10 minutes at $850^{\circ} \mathrm{C}$ ) thick-film system. A first dielectric layer (ESL 4916) with a high thermal expansion was applied first to lower thermal stresses at the interface. This was followed by a second dielectric layer (ESL 4924) featuring better electrical insulation and chemical compatibility with the resistor. Finally, the conductor (ESL 9635B, AgPd) and the piezoresistive (DuPont 2041) compositions were deposited.

In future work, the sensor will be fabricated using a new low-firing and lead-free (including resistors) recently developed thick-film materials system using bismuth-based glasses $[12,13]$. This new system features low-temperature processing $\left(<650^{\circ} \mathrm{C}\right)$, which is very advantageous for piezoresistive sensing as this allows the use of high-strength martensitic stainless steels, lifting an important performance limitation of state-of-the-art thick-film sensors using steel substrates.

\subsection{Characterisation}

The sensor was characterised using a joystick-type setup as depicted in Figure 9, which also constitutes a realistic application setup for wrist rehabilitation exercises. Various combinations of $F_{x}, F_{y}, F_{z}$ forces and $M_{x}$ and $M_{y}$ torques were applied by suspending weights at the tip of the joystick while varying the orientation of the setup and the length of the joystick. The axial torque $M_{z}$ required a slightly different arrangement; in this case, the joystick was horizontal, and the weigths were converted into a pure $M_{z}$ torque using an additional lever and pulley. The resulting bridge signals were measured using an National Instruments 921924 bit acquisition module, with the bridge reconfiguration (see 2.2) being effected by analogue switches.

The signal for the vertical force $F_{z}$ is depicted in Figure 10a; the bridge response at the nominal force (200 N) is around $3.8 \mathrm{mV} / \mathrm{V}$, which compares well with typical ceramic thick-film sensors [14]. From FEM (2.4), we get a strain on the sensing surface of ca. \pm 400 to $\pm 450 \mathrm{ppm}$. Dividing both values yield an estimated longitudinal gauge factor around 9, which agrees well with previous work $[15,16]$. The precision of the measurement for the forces $F_{x}, F_{y}$ and $F_{z}$ at nominal load is between 3 and $4 \%$. The effect of applying a torque $M_{z}$ around the sensor axis is depicted in Figure 10b, and the resulting response at the nominal torque $(5 \mathrm{~N} \bullet \mathrm{m})$ is ca. $0.1 \mathrm{mV} / \mathrm{V}$, a much lower value expected from the dimensioning. The precision on the $M_{z}$ torque is between 5 and $10 \%$, due to the lower sensitivity of the sensor for this DOF.

Given the simplified test jig and unoptimised sensor parameters (see 2.4), these results are encouraging, as they already lie at or below human noticeable levels [17]. Further refinements in sensor design should yield precision better than $2 \%$, in line with our previous results with this technology $[7,8,15]$. 


\section{Conclusion}

A 6 DOF force / torque sensor adapted to wrist rehabilitation has been successfully designed and fabricated using a steel substrate and a commercial high-firing thick-film system. Despite its simple design and unoptimised parameters, it allowed measurement of the forces and torques with acceptable precision. Future work will concentrate on improving the sensor design and switching to a lead-free low-firing thick-film materials system developed in our laboratory. This system, in addition to removing an important environmental concern about the future of this technology, will allow a considerable improvement of performance through the use of high-strength martensitic steels, and possibly some aluminium alloys as well. On the application side, this novel 6DOF sensor will be tested and integrated into a rehabilitation robot to train wrist function, offering new possibilities to assess movement disorder and monitor human-machine interaction.

\section{References}

[1] J. Butterfass, M. Grebenstein, H. Liu, G. Hirzinger, DLR-Hand II: next generation of a dextrous robot hand, Proc. IEEE Int. Conf. Robot. Autom. 1 (2001) 109-114.

[2] L. Dovat, O. Lambercy, R. Gassert, T. Maeder, T. Milner, T.C. Leong, E. Burdet, HandCARE: a cableactuated rehabilitation system to train hand function after stroke, IEEE Trans. Neur. Syst. \& Rehab. Eng. $16(2008) 582-591$

[3] O. Lambercy, L. Dovat, R. Gassert, E. Burdet, T. Chee Leong, T. Milner, A haptic knob for rehabilitation of hand function, IEEE Trans. Neur. Syst. \& Rehab. Eng. 15 (2007), 356-366.

[4] C. Takahashi, L. Der-Yeghiaian, V. Le, R. Motiwala, S. Cramer, Robot-based hand motor therapy after stroke, Brain 131 (2008) 425-437.

[5] D. Diddens, D. Reynaerts, H.V. Brussel, Design of a ring-shaped three-axis micro force/torque sensor, Sensors and Actuators A 46-47 (1995) 225-232.

[6] P.J. Berkelman, L.L. Whitcomb, R.H. Taylor, P. Jensen, A miniature instrument tip force sensor for robot / human cooperative microsurgical manipulation with enhanced force feedback, Lecture Notes in Computer Science 1935 (2004) 897-906.

[7] D. Crottet, T. Maeder, D. Fritschy, H. Bleuler, L.P. Nolte, I.P. Pappas, Development of a force amplitude- and location-sensing device designed to improve the ligament balancing procedure in TKA, IEEE Trans. Biomed. Eng. 52 (2005) 1609-1611. 
[8] D. Crottet, A force-sensing device for assistance in soft-tissue balancing during knee arthroplasty, Thesis $n^{\circ} 3398$ (2005), Ecole Polytechnique Fédérale de Lausanne, Switzerland.

[9] C.F. Yeong, A. Melendez-Calderon, R. Gassert, E. Burdet, ReachMAN: a personal robot to train reaching and manipulation, Proc. IEEE IROS, 2009.

[10] Man-systems integration standards, National Aeronautics and Space Administration (NASA), USA (2008), http://msis.jsc.nasa.gov

[11] J. L. Morse, M.-C. Jung, G. R. Bashford, and M. S. Hallbeck, Maximal dynamic grip force and wrist torque: The effects of gender, exertion direction, angular velocity, and wrist angle, Appl. Ergon. 37 (2006) $737-742$.

[12] C. Jacq, T. Maeder, P. Ryser P, Developpment of low-firing lead-free thick-film materials on steel alloys for piezoresistive sensor applications, Proc. $17^{\text {th }}$ Eur. Microel. \& Packaging Conf. - EMPS2009, Rimini, Italy (2009) P07.

[13] T. Maeder, C. Jacq, C. Grimaldi, P. Ryser, Lead-free low-firing thick-film resistors based on bismuth glasses and ruthenium oxide", Proc., XXXIII Int. Conf. IMAPS Poland Chapter, Gliwice - Pszczyna, Poland (2009) 222-229.

[14] T. Maeder, I. Saglini, G. Corradini, P. Ryser, Low-cost thick-film force sensors for the $100 \mathrm{~N}$ force range, Proc. XXX Int. Conf. IMAPS Poland Chapter, Kraków, Poland (2006) 193-196.

[15] C. Jacq, T. Maeder, P. Ryser, High-strain response of piezoresistive thick-film resistors on titanium alloy substrates, J. Eur. Ceram. Soc. 24 (2004) 1897-1900.

[16] M. Hrovat, D. Belavič, A. Benčan, J. Bernard, J. Holc, J. Cilenšek, W. Smetana, H. Homolka, R. Reicher, L. Golonka, Thick-film resistors on various substrates as sensing elements for strain-gauge applications, Sensors and Actuators A 107 (2003) 261-272.

[17] S. Allin, Y. Matsuoka, R. Klatzky, Measuring just noticeable differences for haptic force feedback: implications for rehabilitation, Proc. 10th IEEE Symp. Haptic Int. Virt. Env. \& Teleop. Syst. (2002) 299302 . 
Thick-film multi-DOF force / torque sensor for wrist rehabilitation

C. Jacq ${ }^{1}$, B. Lüthi ${ }^{1}$, Th. Maeder ${ }^{1}$, O. Lambercy ${ }^{2}$, R. Gassert ${ }^{2}$ and P.Ryser ${ }^{1}$

${ }^{1}$ Laboratoire de Production Microtechnique (LPM), EPFL, Lausanne, Switzerland

${ }^{1}$ Rehabilitation Engineering Lab, ETH Zurich, Zurich, Switzerland

E-mail: caroline.jacq@epfl.ch

Presented at Eurosensors XXIII, manuscript \#8426

\section{TABLES}

Table 1: Specifications of the 6 DOF sensor.

\begin{tabular}{lccc}
\hline Parameter & Symbol & & Specified range \\
\hline Force in x axis & $F_{x}$ & {$[\mathrm{~N}]$} & $0 \ldots 100$ \\
Force in y axis & $F_{y}$ & {$[\mathrm{~N}]$} & $0 \ldots 100$ \\
Force in $\mathrm{z}$ axis & $F_{z}$ & {$[\mathrm{~N}]$} & $0 \ldots 200$ \\
\hline Moment in x axis & $M_{x}$ & {$[\mathrm{~N} \bullet \mathrm{m}]$} & Not specified \\
Moment in y axis & $M_{y}$ & {$[\mathrm{~N} \bullet \mathrm{m}]$} & Not specified \\
Moment in $\mathrm{z}$ axis & $M_{z}$ & {$[\mathrm{~N} \cdot \mathrm{m}]$} & $0 \ldots 5$ \\
\hline
\end{tabular}

Table 2: Dimensions of the sensor \& calculated stresses

\begin{tabular}{lccc}
\hline Parameter & Symbol & & Value \\
\hline Diameter of the device & & {$[\mathrm{mm}]$} & 68 \\
Thickness of the device & & {$[\mathrm{mm}]$} & 7 \\
Distance [centre-axis of the bridge] & & {$[\mathrm{mm}]$} & 13.6 \\
Width of the bridge & $b$ & {$[\mathrm{~mm}]$} & 3.2 \\
Thickness of the bridge & $h$ & {$[\mathrm{~mm}]$} & 0.8 \\
Effective length of the bridge & $l$ & {$[\mathrm{~mm}]$} & 2.1 \\
\hline Stress for $F_{x}=100 \mathrm{~N}$ (eq. 1) & $\sigma_{/ /}$ & {$[\mathrm{MPa}]$} & 20 \\
Stress for $F_{z}=70 \mathrm{~N}$ (eq. 2$)$ & $\sigma_{b}$ & {$[\mathrm{MPa}]$} & 108 \\
\hline
\end{tabular}

Table 3: Nominal and design forces (F) / torques (M). Nominal values are estimated for wrist function, and design ones are taken with $100 \mathrm{MPa}$ calculated stress in the measuring structures.

\begin{tabular}{cccc}
\hline Axis & Nominal & Design & \\
\hline$F_{z}$ & 200 & 200 & {$[\mathrm{~N}]$} \\
$F_{x}, F_{y}$ & 100 & 1000 & {$[\mathrm{~N}]$} \\
\hline$M_{z}$ & 5 & 20 & {$[\mathrm{~N} \cdot \mathrm{m}]$} \\
$M_{x}, M_{y}$ & Not specified & 3 & {$[\mathrm{~N} \cdot \mathrm{m}]$} \\
\hline
\end{tabular}




\section{Figures}

Figure 1: $2 \mathrm{DOF}$ robotic device developed for the rehabilitation of hand function after stroke, the Haptic Knob [3].

Figure 2: Schematic arrangement of the sensitive modules.

Figure 3: Bridge in tension / compression for horizontal forces $(\mathrm{H})$ and in bending for vertical forces (V), with corresponding Wheatstone bridge configurations.

Figure 4: Layout of reconfigurable Wheatstone circuit.

Figure 5: Cross section of a part of a bridge.

Figure 6: $3 \mathrm{D}$ view of the sensor and detailed view of the bridges.

Figure 7: FEM analysis (Von Mises stress), for applied (a) $5 \mathrm{~N} \cdot \mathrm{m} M_{z}$ torque; (b) applied $200 \mathrm{~N} F_{z}$ force, scale of deformations: 500 .

Figure 8: Photograph of the final device.

Figure 9: Sensor in the setup

Figure 10: Signal for applied vertical force $F_{z}$ (a) and moment $M_{z}$ (b). 


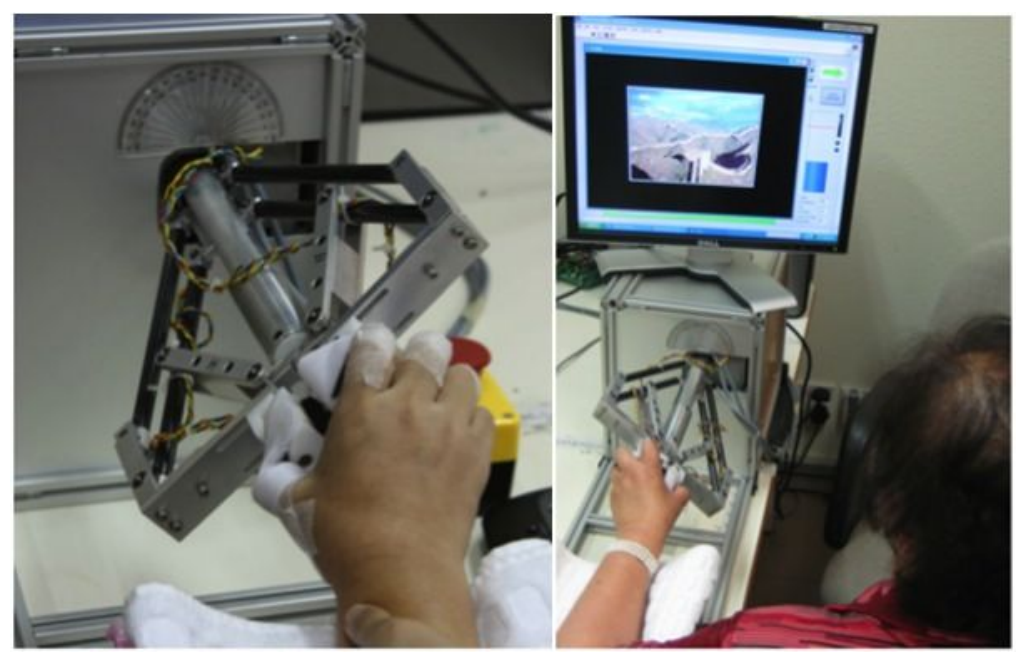

Figure 1: 2DOF robotic device developed for the rehabilitation of hand function after stroke, the Haptic Knob [3].

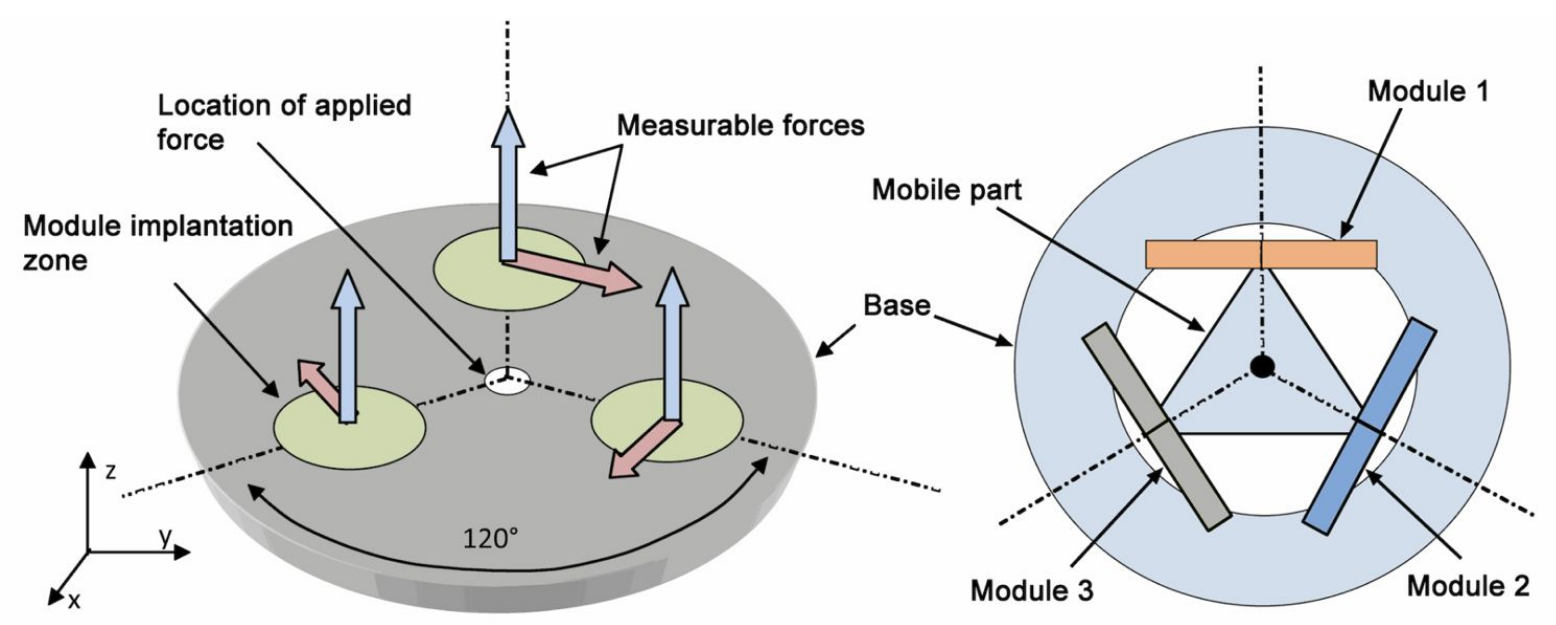

Figure 2: Schematic arrangement of the sensitive modules.

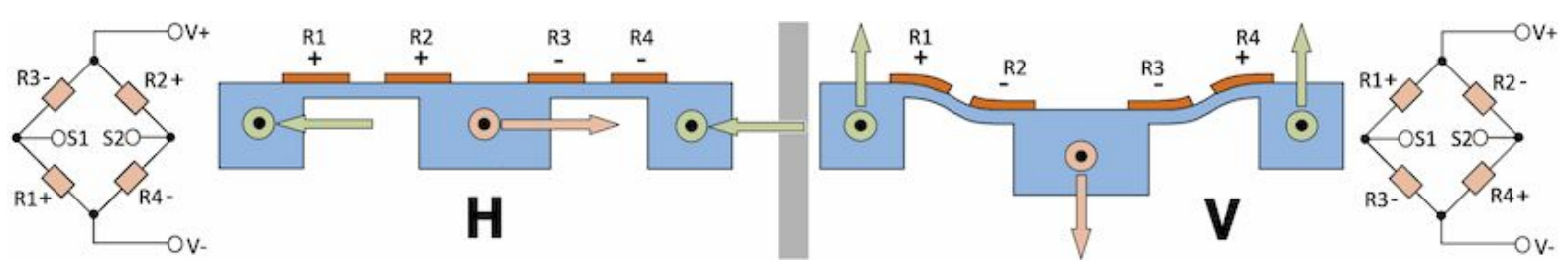

Figure 3: Bridge in tension / compression for horizontal forces $(\mathrm{H})$ and in bending for vertical forces (V), with corresponding Wheatstone bridge configurations. 


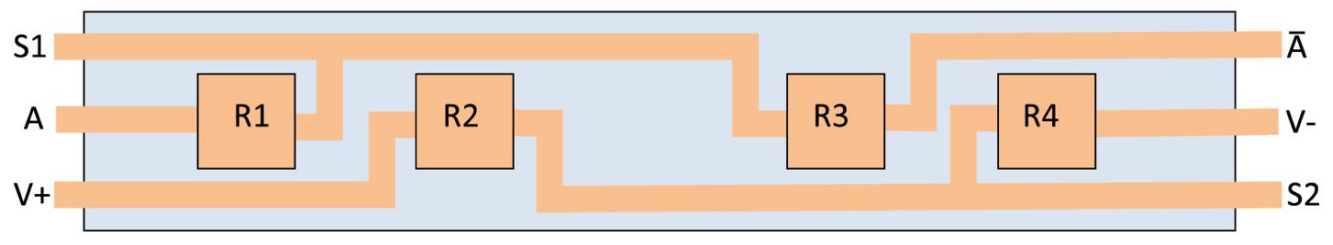

Figure 4: Layout of reconfigurable Wheatstone circuit.

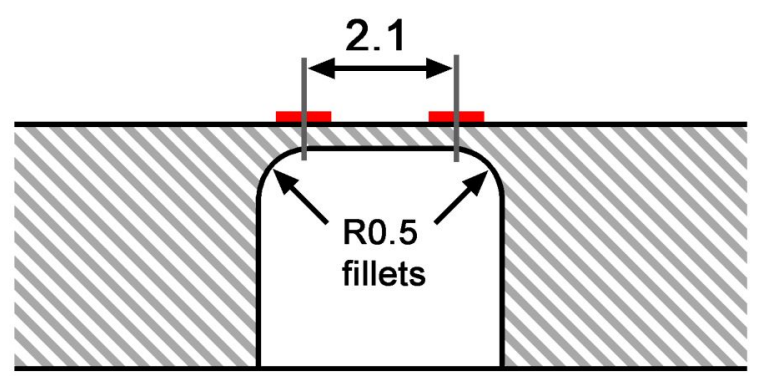

Figure 5: Cross section of a part of a bridge.
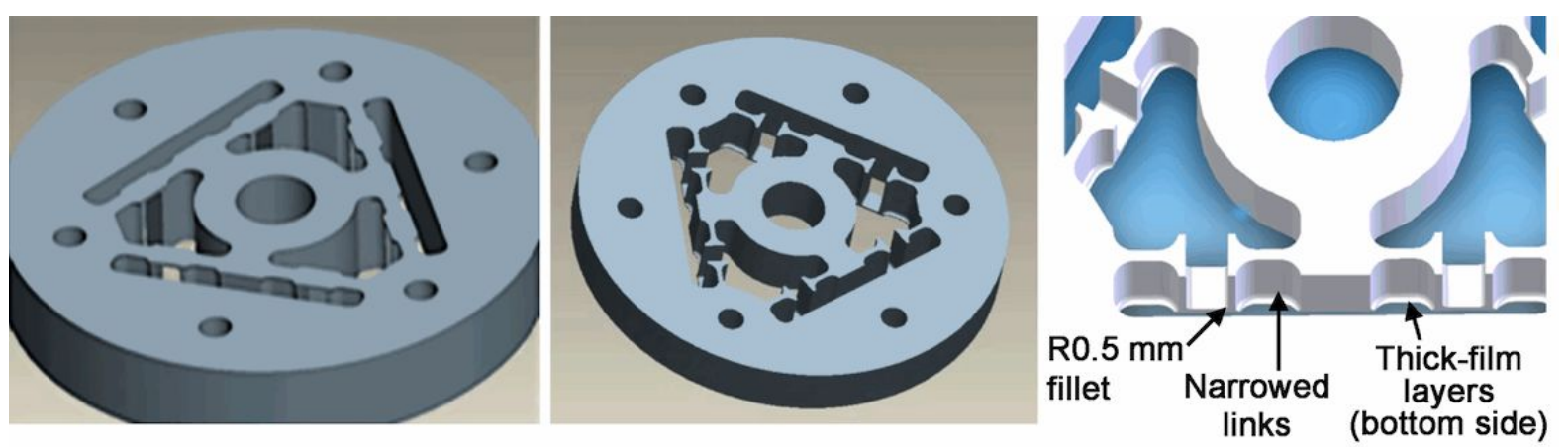

Figure 6: 3D view of the sensor and detailed view of the bridges.
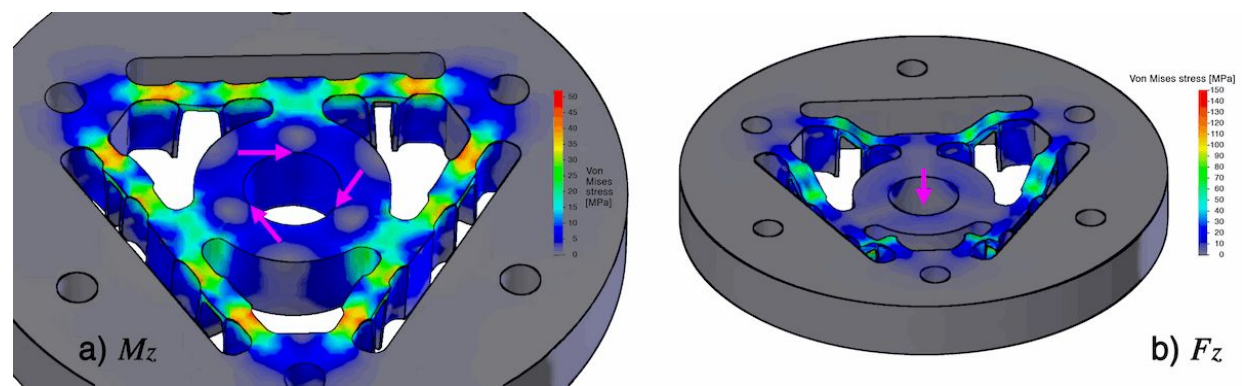

Figure 7: FEM analysis (Von Mises stress), for applied (a) $5 \mathrm{~N} \cdot \mathrm{m} M_{z}$ torque; (b) applied $200 \mathrm{~N} F_{z}$ force, scale of deformations: 500 . 


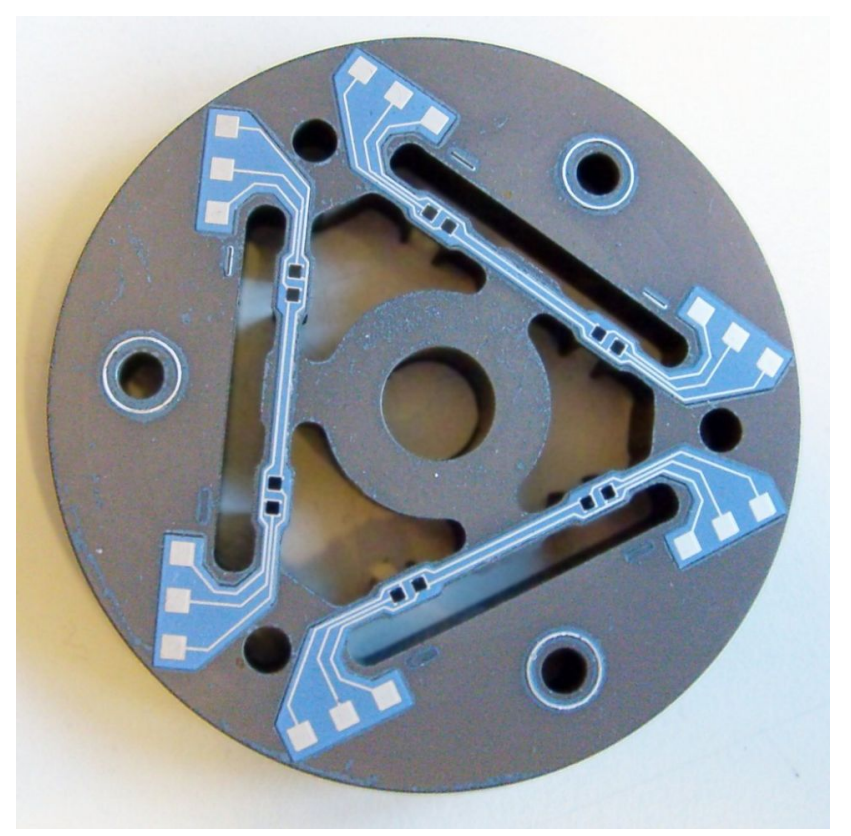

Figure 8: Photograph of the final device.

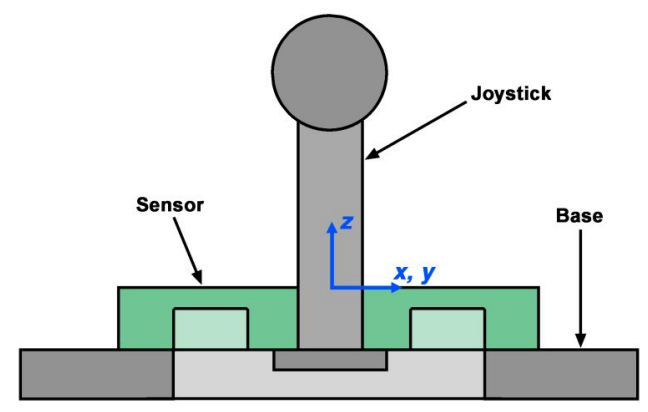

Figure 9: Sensor in the setup
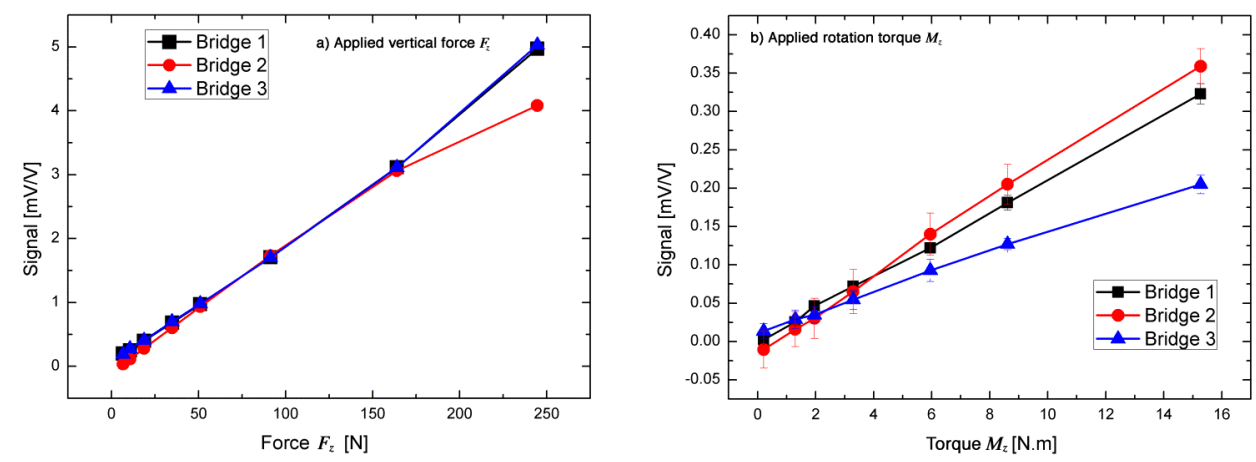

Figure 10: Signal for applied vertical force $F_{z}$ (a) and moment $M_{z}(\mathrm{~b})$. 


\section{Biographies}

Caroline Jacq graduated with a Master thesis in Materials Science from the Institut Supérieur des Matériaux du Mans (ISMANS), and is working as a researcher at the Ecole Polytechnique Fédérale de Lausanne (EPFL) since 2001. Her main research interests concern the development and study of novel lead-free thick-film materials systems and their application to medical devices.

Barthélémy Lüthi is a Master student in Microtechnology at the Ecole Polytechnique Fédérale de Lausanne (EPFL). He is currently carrying out his Master thesis at Berkeley University, and will gain his Master degree in 2010, with a specialization in Production Techniques and Robotics.

Thomas Maeder pursued with a $\mathrm{PhD}$ in piezoelectric thin films, and a post-doc at IBM Rüschlikon in single-crystal conductive oxides, after graduating from the Ecole Polytechnique Fédérale de Lausanne (EPFL) in materials science. He now heads the thick-film technology group at the EPFL, where current areas of interest are thick-film and LTCC technology for advanced sensor and packaging applications, and sensor networking.

Peter Ryser received a Master degree in Physics (University Neuchâtel 1979), a PhD in applied Physics (University Geneva 1985 ) and a Masters Degree in Corporate Management (Lucerne 1993). His professional background includes several R\&D activities. From 1990-1998 he was the head of research at Siemens Building Technologies. Since 1999 Peter Ryser is Professor at the Swiss Federal Institute of Technology EPFL in Lausanne and act as a director for the micro engineering section.

Olivier Lambercy was born 1982 in Vevey, Switzerland. He studied Microengineering at the Ecole Polytechnique Fédérale de Lausanne (EPFL), Switzerland, and is currently completing his PhD at the National University of Singapore (NUS). During his $\mathrm{PhD}$, he investigated robot-assisted rehabilitation of hand function after stroke. In January 2009, he joined the Rehabilitation Engineering Lab, where he continues his research on robot-assisted hand rehabilitation and the use of haptic devices for medical applications.

Roger Gassert has been assistant professor of rehabilitation engineering at ETH Zürich since December 2008. Born in 1976 in Solothurn, Switzerland, he received the M.Sc. degree in microengineering and the Ph.D. degree in robotics and neuroscience from the Ecole Polytechnique Fédérale de Lausanne (EPFL), Switzerland in 2002 and 2006, respectively. From December 2007 to November 2008 he headed the joint robotics lab between EPFL and the University of Tokyo at the Robotic Systems Lab at EPFL. His research interests are in rehabilitation- and medical robotics, haptics, assistive devices and neuroscience. 